Central Washington University

ScholarWorks@CWU

All Faculty Scholarship for the College of the Sciences

6-15-1993

Self-consistent determination of electronic structure and elementary excitations of finite modulation-doped superlattices

Roger H. Yu

Follow this and additional works at: https://digitalcommons.cwu.edu/cotsfac

Part of the Atomic, Molecular and Optical Physics Commons, and the Quantum Physics Commons 


\title{
Self-consistent determination of electronic structure and elementary excitations of finite modulation-doped superlattices
}

\author{
Roger H. Yu \\ Department of Physics, Central Washington University, Ellensburg, Washington 98926
}

(Received 26 August 1992)

\begin{abstract}
A self-consistent framework for the study of the electronic level structure of finite superlattices has been proposed. One of the surface states (Tamm states) found in our calculation crosses the Fermi energy in the energy gap when the depletion effect near the surface increases. We have shown the existence of low-energy Tamm states in our calculation when the surface barrier is lower than that of the interior. The electronic excitations of the superlattice have been studied via the electron-energy-loss function within the random-phase approximation. Two plasmon modes (well above the phonon response frequency) due to the Tamm states have also been found. We believe these plasmon modes can be observed by high-resolution electron-energy-loss spectroscopy.
\end{abstract}

\section{INTRODUCTION}

Recent advances in molecular-beam epitaxy have allowed the growth of high-quality $\mathrm{GaAs} / \mathrm{Al}_{x} \mathrm{Ga}_{1-x} \mathrm{As}$ superlattices with abrupt interfaces, similar lattice structure, and matching lattice parameters. ${ }^{1}$ These developments in material fabrication have generated much interest in the physical properties of superlattices. The electronic miniband structure has been extensively investigated following Dingle, Gossard, and Wiegmann, ${ }^{2}$ who carried out optical-absorption measurements on a onedimensional $\mathrm{GaAs} / \mathrm{Al}_{x} \mathrm{Ga}_{1-x} \mathrm{As}$ superlattice. The single-particle and collective excitations in a multiquantum-well system have been studied by Pinczuk ${ }^{3}$ and Ploog ${ }^{4}$ and co-workers via inelastic light scattering. In these experiments, discrete plasmon modes in a layered two-dimensional (2D) electron gas predicted by Giuliani and Quinn ${ }^{5}$ were observed. Recently, in the quantum Hall effect (QHE) measurement done by Störmer et al. ${ }^{6}$ and in the optical spectroscopies performed by Ohno et al.,$^{7}$ surface localized electronic states (also known as Tamm states ${ }^{8}$ ) in a GaAs $/ \mathrm{Al}_{x} \mathrm{Ga}_{1-x} \mathrm{As}$ semiconductor superlattice which resulted in the inclusion of the surface effect were observed. On the theoretical side, the miniband structure of a $\mathrm{GaAs} / \mathrm{Al}_{x} \mathrm{Ga}_{1-x}$ As superlattice with cyclic boundary conditions has been studied using a variety of methods, summarized by Bastard, Brum, and Ferreira, ${ }^{1}$ including the Kronig-Penney model, the envelope-function framework, 9,10 and the tight-binding approximation. ${ }^{11}$ However, real superlattices consist of only a finite number of alternating layers of different materials, and so it is essential that the electronic states localized at the surface be included in a detailed electronic structure calculation. Zhang and Ulloa $^{12}$ have included surface effects in their calculations based on a tightbinding envelope-function approximation and have found surface states lying in the miniband gap. A terminated Kronig-Penney model has been used for studying electronic surface states in compositional superlattice by Steslicka, Kocharczyk, and Glasser, ${ }^{13}$ Bloss, ${ }^{14}$ and Tikhodeev. ${ }^{15}$ Zhang and Ulloa also presented a theoretical study of the far-infrared optical response of a doped su- perlattice in the long-wavelength limit $(Q=0)$ (Refs. 16 and 17) using the $d$-parameter formalism. ${ }^{18}$ Both intraminiband and interminiband plasmons as well as surface modes related to charge depletion in the superlattice were predicted in their calculations. The surface states found by Zhang and co-workers ${ }^{12,16,17}$ and in other recent model calculations ${ }^{19}$ exist in the gap, well above the highest occupied miniband.

In this paper, we report results found using the self-consistent calculations of the conduction-band structure of an inhomogeneously doped tunneling $\mathrm{GaAs} / \mathrm{Al}_{x} \mathrm{Ga}_{1-x} \mathrm{As}$ superlattice of a finite number of layers, in which the electron gases are also strongly anisotropic and inhomogeneous. In our self-consistent procedure for solving the Schrödinger and Poisson equations, we introduced a positive nonuniform jellium background, which incorporates the alternating square-wavelike donor distribution in the superlattice. This approach is similar to that of introducing atoms in jellium models. Second, we suggest the use of high-resolution electronenergy-loss spectroscopy to probe the elementary excitations due to the Tamm states we obtained, because the penetration depth of the incident electron beam, a few hundred angstroms, is comparable with the spatial localization of the surface states. Our theory of the elementary excitations in the superlattice is based on the selfconsistent computation of the dynamical response function at finite wave vector $(Q \neq 0)$. The associated energyloss function predicts the dispersion relations of the excitation modes. The calculations are carried out within the random-phase-approximation (RPA), and represent a generalization of the early work performed by Ehlers and Mills ${ }^{20-22}$ and by the present author and Hermanson ${ }^{23,24}$ that concentrated on electron energy losses from accumulation layers and depletion layers at the surface on an (otherwise) homogeneous semiconductor. To the best of our knowledge, the calculation presented in this paper is the first realistic theory of the ground-state properties and the dynamical response of electrons in a finite superlattice, which considers all of the significant effects simultaneously.

In what follows we present fully self-consistent calculations of the electronic level structure in semiconductor 
superlattices and the dynamical response of electron gases to an external probe. Section II summaries our self-consistent procedure of solving Schrödinger and Poisson's equations. The nonlocal response formalism to our model system is given in Sec. III. In Sec. IV, we discuss the results of both ground-state and dynamical response calculations. Section $\mathrm{V}$ contains our summary and conclusions.

\section{ELECTRONIC LEVEL STRUCTURE}

Our structural model of an $N$-layer-modulation-doped $\mathrm{GaAs} / \mathrm{Al}_{x} \mathrm{Ga}_{1-x}$ As superlattice is depicted in Fig. 1(a) by the broken lines (dotted and dashed lines for interior and surface, respectively). The sample is bounded by two infinitely hard potential walls on both sides. As discussed
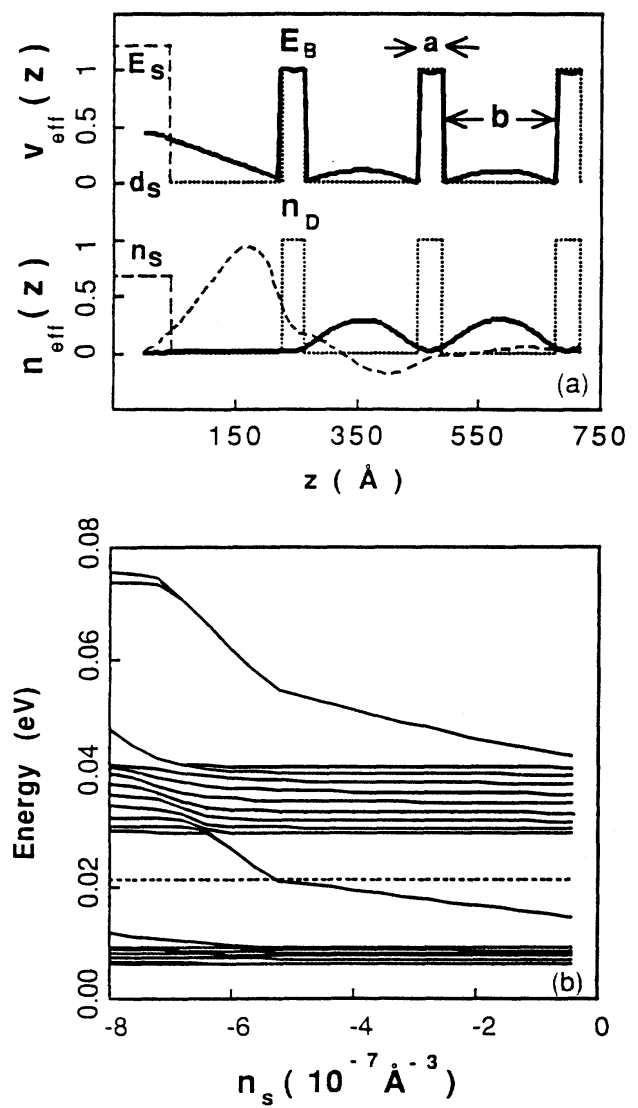

FIG. 1. (a) The calculated self-consistent charge density and effective potential (solid lines, showing only the first three layers). The background potential and doping density profiles of the superlattice interior are depicted schematically by the dotted lines. The long-dashed lines represent the surface (internal) barrier and surface charge density. The short-dashed line is the surface-state wave function. The sample parameters used in the calculation are the following: 11 layers, $a=38 \AA ; b=188 \AA$; $E_{B}=0.134 \mathrm{eV} ; n_{D}=1.0 \times 10^{-6} \AA^{-3} ;$ surface barrier width $d_{s}=100 \AA ; E_{s}=0.2 \mathrm{eV} ; n_{s}=-4.5 \times 10^{-7} \AA^{-3} ; m^{*}=0.067 \mathrm{~m}$; and $\varepsilon=12.9$. One hundred (100) sine waves were used to expand the eigenfunction $\phi_{i}$. (b) The self-consistent energy levels as a function of surface charge density. The dashed line shows the Fermi energy. in Ref. 20, the latter boundary condition is appropriate because the penetration depth of the conduction-band states into the surface barrier is only a few angstroms. The background potential barrier height and the doping density for the interior layers are denoted by $E_{B}$ and $n_{D}$, respectively. The surface barrier height $E_{s}$ and the doping density $n_{s}$, which simulates the depletion (negative $n_{s}$ ) and accumulation (positive $n_{s}$ ) effects, is generally different from that of interior layers. Our self-consistent procedure for obtaining the conduction-band structure may be outlined as follows. The conduction electrons inside the quantum wells are treated as a free-electron gas, in the plane parallel to the surface, and are characterized by a position-independent effective mass $m^{*} \cdot{ }^{25}$ In the effective-mass approximation, the electron eigenfunction has the form

$$
\varepsilon_{i}\left(\mathbf{K}_{\|}\right)=\frac{\hbar^{2} \mathbf{K}_{\|}^{2}}{2 m^{*}}+\varepsilon_{i},
$$

where the first term describes the energies of free-electron motion parallel to the surface, and the second term $\varepsilon_{i}$ represents the energy levels of the quantized motion of electron along the direction of growth. $\varepsilon_{i}$ and corresponding single-particle wave functions satisfy the Schrödinger equation,

$$
\left[\frac{\hbar^{2}}{2 m^{*}} \frac{d^{2}}{d z^{2}}+v_{\mathrm{eff}}(z)\right] \phi_{i}(z)=\varepsilon_{i} \phi_{i}(z) .
$$

The effective potential $v_{\text {eff }}(z)$ in the Schrödinger equation has the following three components: (1) The background potential inside the superlattice $v_{B}(z)$, shown by the dotted and dashed lines in the top panel of Fig. 1(a),

$$
\begin{aligned}
v_{B}(z)= & E_{s}\left[u(z)-u\left(z-d_{s}\right)\right] \\
& +E_{s}\left(u\left\{z-\left[d_{s}+b+(n-1) c\right]\right\}\right) \\
& +\sum_{i=1}^{N-1} E_{b}\left(u\left\{z-\left[d_{s}+b+(i-1) c\right]\right\}\right. \\
& \left.-u\left[z-\left(d_{s}+b+i c\right)\right]\right),
\end{aligned}
$$

where $N$ is the number of periods, $d_{s}$ is the surface potential barrier width, $b$ is the potential well width, $c$ is width of a period, and $u(z)$ is the unit step function; (2) the exchange and correlation potential $v_{\mathrm{xc}}(z)$ of Hedin and Lundqvist; ${ }^{26}$ and (3) the Hartree potential $v_{H}(z)$, which is related to the charge density by Poisson's equation,

$$
v_{H}(z)=-\frac{2 \pi e^{2}}{\varepsilon} \int_{0}^{L}\left[n\left(z^{\prime}\right)-n_{D}\left(z^{\prime}\right)\right]\left|z-z^{\prime}\right| d z,
$$

where $L$ is the sample thickness and $n_{D}(z)$ is the donor density as a function of position $z$,

$$
\begin{aligned}
n_{D}(z)= & n_{s}\left[u(z)-u\left(z-d_{s}\right)\right] \\
& +n_{s}\left(u\left\{z-\left[d_{s}+b+(n-1) c\right]\right\}\right) \\
& +\sum_{i=1}^{N-1} n_{D}\left(u\left\{z-\left[d_{s}+b+(i-1) c\right]\right\}\right. \\
& \left.-u\left[z-\left(d_{s}+b+i c\right)\right]\right),
\end{aligned}
$$


depicted by the dotted and dashed lines in the lower portion of Fig. 1(a). The electron density $n(z)$ is related back to the eigenfunctions $\phi_{i}(z)$ through the Fermi-Dirac distribution function. The solutions of the Schrödinger equation $\phi_{i}(z)$ are used to calculate $n(z)$, and to build $v_{H}(z)$ and $v_{\mathrm{xc}}(z)$. The Schrödinger equation is solved iteratively until self-consistency is reached. The Fermi energy is determined by the condition of overall charge neutrality of the sample. ${ }^{24}$

In our numerical simulation, much care has been taken in choosing the number of sine waves used in the eigenfunction expansion. We have found that taking 100 sine waves leads to convergence of the final results. We have also found that it is necessary to have about 300 iterations to obtain convergence: then the Fermi energy difference between consecutive iterations is no greater than $10^{-6} \mathrm{meV}$.

\section{NONLOCAL RESPONSE FORMALISM WITHIN THE RANDOM-PHASE APPROXIMATION}

The dynamical response of the electron system is described by the RPA formalism. We compute the energyloss function $P\left(q_{\|}, \omega\right)$ (Refs. 20-24) corresponding to energy and wave vector transfers $h \omega$ and $q_{\|}$, respectively, to obtain an explicit expression for the cross section of inelastic electron scattering.

The electron-energy-loss function in the dipole approximation is given by

$$
\begin{aligned}
P\left(q_{\|}, \omega\right)=\frac{8 e^{2}}{|1+\varepsilon|^{2}} \int_{0}^{L} d z \int_{0}^{L} d z^{\prime} e^{-q_{\|}\left(z+z^{\prime}\right)} & \\
& \times \operatorname{Im}\left[\chi\left(q_{\|}, \omega ; z, z^{\prime}\right)\right],
\end{aligned}
$$

where the density response function $\chi\left(q_{\|}, \omega ; z, z^{\prime}\right)$ for the interacting electrons satisfies an integral equation in the random-phase-approximation

$$
\begin{array}{rl}
\chi\left(q_{\|}, \omega ; z, z^{\prime}\right)= & \chi^{0}\left(q_{\|}, \omega ; z, z^{\prime}\right) \\
-\int_{0}^{L} d z_{1} \int_{0}^{L} & d z_{2} \chi^{0}\left(q_{\|}, \omega ; z, z_{1}\right) \\
& \times v\left(q_{\|} ; \omega ; z_{1}, z_{2}\right) \\
& \times \chi\left(q_{\|}, \omega ; z_{2}, z^{\prime}\right),
\end{array}
$$

where $v\left(q_{\|}, \omega ; z_{1}, z_{2}\right)$ is the Fourier transform of the electron-electron interaction including the Hartree as well as the exchange-correlation potential, and $\chi^{0}\left(q_{\|}, \omega ; z, z^{\prime}\right)$ is the response function of the noninteracting electron gas, which can be expressed in terms of wave functions $\phi_{l}(z)$,

$\chi^{0}\left(q_{\|}, \omega ; z, z^{\prime}\right)=\sum_{i, j} \Pi_{i j}\left(q_{\|}, \omega\right) \phi_{i}(z) \phi_{i}\left(z^{\prime}\right) \phi_{j}(z) \phi_{j}\left(z^{\prime}\right)$,

where

$$
\Pi_{i j}\left(q_{\|}, \omega\right)=\int \frac{d^{2} \mathbf{k}_{\|}}{(2 \pi)^{2}} \frac{f\left(\varepsilon_{\mathbf{k} \| i}\right)-f\left(\varepsilon_{\mathbf{k}_{\|}}+\mathbf{q}_{\|, j}\right)}{\varepsilon_{\mathbf{k}_{\|}+\mathbf{q}_{\|, j}}-\varepsilon_{\mathbf{k}_{\|, i}}+i \eta} .
$$

In calculating the noninteracting response function, a phenomenological damping constant $\eta \sim 10^{-5} \mathrm{meV}$ has been used. Then the loss function is essentially independent of the constant $\eta$.
To discretize the integral equation for $\chi\left(q_{\|}, \omega ; z, z^{\prime}\right)$ [Eq. (7)], the double-cosine series representation is used, ${ }^{27}$ $\chi\left(q_{\|}, \omega ; z, z^{\prime}\right)=\sum_{i, j} \chi_{i j}\left(q_{\|}, \omega\right) \cos \left(\frac{i \pi z}{L}\right) \cos \left(\frac{j \pi z^{\prime}}{L}\right)$,

to transform Eq. (7) into the matrix equation

$$
\begin{aligned}
\chi_{i j}\left(q_{\|}, \omega\right)= & \chi_{i j}^{0}\left(q_{\|}, \omega\right) \\
& -\sum_{i^{\prime} j^{\prime}} \chi_{i i^{\prime}}^{0}\left(q_{\|}, \omega\right) v_{i^{\prime} j^{\prime}}\left(q_{\|}, \omega\right) \chi_{j j^{\prime}}\left(q_{\|}, \omega\right) .
\end{aligned}
$$

The matrix element $v_{i j}\left(q_{\|}, \omega\right)$ is the double-cosine Fourier transform of the electron-electron interaction in the superlattice, and $\chi_{i j}^{0}\left(q_{\|}, \omega\right)$ is defined as

$$
\chi_{i j}^{0}\left(q_{\|}, \omega\right)=\frac{\mu_{i} \mu_{j}}{L^{2}} \sum_{k l} \Pi_{k l}\left(q_{\|}, \omega\right) W_{k l}^{i} W_{k l}^{j},
$$

where $\Pi_{k l}\left(q_{\|}, \omega\right)$ is given by Eq. (9); $W_{k l}^{m}$ and $\mu_{i}$ are given by

$$
\begin{aligned}
& W_{k l}^{m}=\frac{1}{2} \sum_{k^{\prime} l^{\prime}} b_{k k^{\prime}} b_{11^{\prime}}\left(\delta_{m, k^{\prime}-1^{\prime}}+\delta_{m, l^{\prime}-k^{\prime}}+\delta_{m, k^{\prime}+1^{\prime}}\right), \\
& \mu_{i}= \begin{cases}1, & i=0 \\
2, & i \geq 0 .\end{cases}
\end{aligned}
$$

\section{RESULTS AND DISCUSSIONS}

The first system we consider is the sample used in the quantum Hall effect (QHE) experiment performed by Störmer et al. ${ }^{6}$ The calculated self-consistent potential and charge density, with sample parameters chosen to match the experiment, are shown by the solid lines in Fig. 1(a). Fermi-level pinning below its position in intrinsic GaAs is introduced by an assumed negative surface charge density $n_{s}$ in the GaAs buffer layer. At $n_{s}=-4.5 \times 10^{-7} \AA,^{-3}$ which places the pinning level $750 \mathrm{meV}$ below the conduction band, the conductionband edges in the wells and barriers are bent, and the electron charge density in the first well is almost completely depleted. Because of the thin lower barrier between the wells, the charge density shown in Fig. 1(a) is an anisotropic three-dimensional electron gas. The charge depletion effect extends three layers into the surface. With the chosen sample parameters, the calculations predict a surface state whose wave function is highly localized in the first layer [short-dashed line in the bottom panel of Fig. 1(a)] and vanishes within three wells. The miniband width remains constant with respect to the surface charge density $n_{s}$ as shown in Fig. 1(b). The miniband results of our self-consistent calculation are as follows: the width of the lowest band $\Delta_{1}=0.003 \mathrm{eV}$, the energy gap $E_{\text {gap }}=0.023 \mathrm{eV}$, the width of the second bandwidth $\Delta_{2}=0.012 \mathrm{eV}$, and the Fermi energy $E_{F}=0.013 \mathrm{eV}$ above the lowest miniband; there are three minibands below the barrier. All the calculated results agree very well with the experimental data. Most interestingly, the calculated surface state (Tamm state) splits off the top of the lowest miniband, crosses the Fer- 
mi energy and eventually joins the upper miniband as the negative surface charge is increased in magnitude. Although our calculations were performed for the case of zero magnetic field, the results can still provide a qualitative explanation for the puzzling small experimental activation energy $\Delta_{\text {exp }}=0.26 \mathrm{meV}$ because at finite magnetic field, the surface localized state would also cross the Fermi energy at some surface charge density $n_{s}$ so that the energy difference $\Delta E$ between the Fermi level and the eigenstate can be small. It is worth noting that the energy of the Tamm state for the accumulation layer (positive surface charge $n_{s}$ ) is lower than the lowest miniband. We note also that the surface state crosses the upper miniband and that anticrossing behavior occurs. Our calculated wave function (not shown here) at $n_{s}=-7.0 \times 10^{-7}$ $\AA^{-3}$ is a superposition of surface and bulk states, confirming that the mixing occurs inside the miniband. ${ }^{28}$

Figures 2(a) and 2(b) show the self-consistent energy levels as a function of potential well width and doping density, respectively, for an accumulation layer $n_{s}=0.2 \times 10^{-4} \AA^{-2}$, and the other parameters chosen to match the experiment. ${ }^{6}$ The energy levels versus well width curve is similar to that obtained by Bloss ${ }^{14}$ using a terminated Kronig-Penney model. For small well width, all the energy levels are approximately evenly spaced. When the well width is increased, the barrier width is decreased relatively, and the interaction between the Tamm
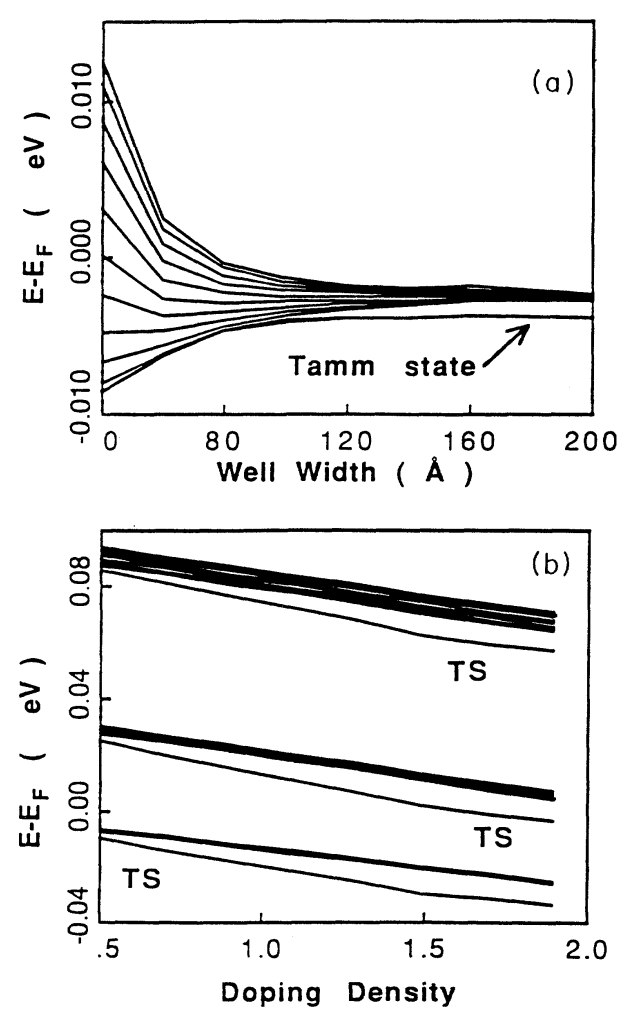

FIG. 2. (a) and (b) show the energy levels against well width and doping density, respectively, for positive surface charge density $n_{s}=0.2 \times 10^{-4} \AA^{-2}$. The other parameters are the same as in Fig. 1. The doping density is given in units of $10^{-6} \AA^{-3}$. state and the extended states of the superlattice strengthens. For well width larger than $100 \AA$, the interaction is so strong that the Tamm state emerges from the lower limit of the miniband. Increasing in doping density also strengthens the interaction between the surface state and the minibands. As doping density increases, the surface-state energy is pushed downward from the miniband, as shown in Fig. 2(b), and the whole energy spectra are lowered almost linearly in order for the system to reach self-consistency.

We also consider the system investigated by Ohno et al., ${ }^{7}$ in which an unusual surface barrier called the "internal surface" was formed inside the semiconductor surface by an $\mathrm{Al}_{x} \mathrm{Ga}_{1-x}$ As terminating layer with elevated Al content compared with that in the interior superlattice barriers, such that the corresponding barrier is $\Delta E=E_{S}-E_{B}$ higher than those in the rest of the superlattice. A "high-energy" Tamm state, appearing above the lowest miniband, was observed in their photoluminescence (PL) spectra. Figure 3 shows our calculated selfconsistent results for the energy levels of the model superlattice; all the parameters were chosen to match experiment. $^{7}$ If $\Delta E>0$, the high-energy Tamm state detaches from the miniband, in exact agreement with the observation made by Ohno et al. ${ }^{7}$ The calculated band width is $0.042 \mathrm{eV}$ and is independent of $\Delta E$. For $\Delta E=0.755 \mathrm{eV}$, the energy difference between the Tamm state and the bottom of the miniband is $0.05 \mathrm{eV}$, in excellent agreement with the measured value, $0.049 \mathrm{eV}$. The anticrossing behavior is also exhibited in Fig. 3. Note that the Tamm state lies below the miniband if $\Delta E<0$, as shown in Fig. 3. Actually, there coexist two more Tamm states in the gap between the first and second minibands as displayed by the inset of Fig. 3. In the experiment, the internal surface barrier can be lowered by reducing the $\mathrm{Al}$ content in the $\mathrm{Al}_{x} \mathrm{Ga}_{1-x} \mathrm{As}$ terminating layer. A similar "lowenergy" Tamm state had been observed by Agulla-Rueda et al. $;^{29}$ in their case the last GaAs well was widened.

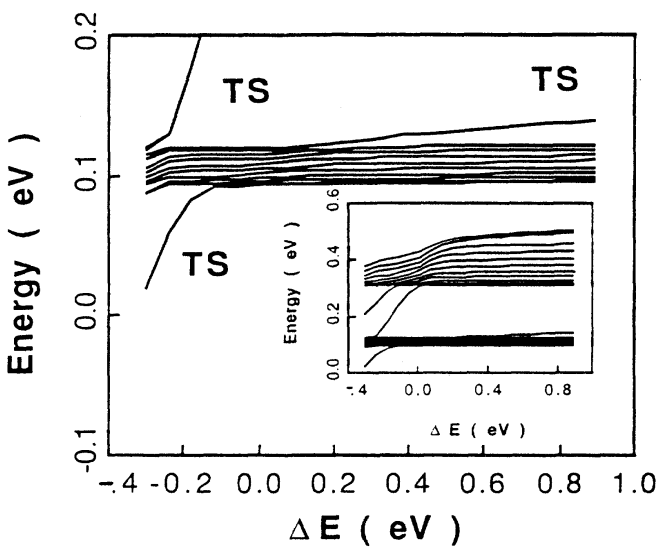

FIG. 3. Calculated energy levels as a function of $\Delta E$, the difference between the surface barrier height and that of the rest of the superlattice, with $d_{s}=100 \AA, a=b=40 \AA$, $n_{D}=1.0 \times 10^{-8} \AA^{-3}, n_{s}=0$ (no surface charge), $E_{B}=0.3 \mathrm{eV}$, and the other parameters are the same as used in Ref. 7. The inset shows the same electronic band structure but on a larger energy scale. 
We have also performed self-consistent calculations without taking into account the exchange and correlation potential $v_{\mathrm{xc}}(z)$ and have found that the exchange and correlation potential does not affect the subband structure of our model superlattice significantly. The results without exchange and correlation potential for the system shown in Fig. 1 are following: the lowest subband energy is raised by $2.3 \mathrm{meV}$, the Fermi energy is lowered by $0.01 \mathrm{meV}$, the miniband width and the miniband gap remain unchanged, the peak position of the wave function, shown by the dashed line in Fig. 1, is shifted to the left by $0.7 \AA$, and the shape of the charge density also remains the same. Therefore, the approach employed here in our calculations is effectively the same as the Hartree approximation which is consistent with the RPA for the dynamics of the electron gas. Recently, the localdensity approximation (LDA) itself and the use of the combination of the LDA and RPA calculations of the excitations have been challenged by several authors (e.g., Gammon et al. ${ }^{30}$ and Brey, Johnson, and Halperin ${ }^{31}$ ). However, in our particular case, we have not encountered such a difficulty because of the weakness of the exchange and correlation potential in the superlattice system under consideration.

We now turn our attention to the dynamical response of the superlattice to an external probe. At low temperature, the Tamm state below the miniband is more heavily populated than that in the Tamm state above the miniband; therefore, the former state should generate a stronger signal in the energy-loss function. For a doping density $n_{D}=1.9 \times 10^{-6} \AA^{-3}$, barrier height $E_{B}=0.4 \mathrm{eV}$, surface barrier $E_{s}=0.2 \mathrm{eV}$ (lower than that of the interior superlattice), and other parameters the same as given in Fig. 1(a), the miniband structure is described as the following: the widths of the lowest two minibands are 0.001 and $0.003 \mathrm{eV}$, respectively, the band gap is $0.029 \mathrm{eV}$, and the Fermi level is just below the bottom of the second miniband. Based on the ground-state properties of the system, we have calculated electron-energy-loss function $P\left(q_{\|}, \omega\right)$. Some representative results for both accumulation layer $\left(n_{s}=0.2 \times 10^{-4} \AA^{-2}\right)$ and depletion layer $\left(n_{s}=-0.4 \times 10^{-4} \AA^{-2}\right)$ are plotted in Figs. 4 and 5 , respectively. For both accumulation and depletion layers the miniband structure remains the same, but the corresponding Tamm states are different; the lowest Tamm state for the accumulation case exists $0.007 \mathrm{eV}$ below the lowest miniband while the Tamm state for the depletion case stays $0.01 \mathrm{eV}$ above it. The loss functions shown in the left column in Figs. 4 and 5 are calculated by the density response function $\chi^{0}\left(q_{\|}, \omega ; z, z^{\prime}\right)$ for noninteracting electron gas and they represent the spectra of singleparticle excitations. The collective excitation spectra calculated using $\chi\left(q_{\|}, \omega ; z, z^{\prime}\right)$ are shown in the right column in Figs. 4 and 5. For the smallest wave vector $q_{\|}=0.0003 \AA^{-1}$, an intraminiband single-particle excitation $(\omega \sim 0)$, associated with the lowest subband, and four interminiband single-particle excitations between the lowest minibands $(\omega \sim 0.03 \mathrm{eV} \sim$ band gap $=0.029 \mathrm{eV})$ can be seen in Fig. 4. The peak near $\omega=0.09 \mathrm{eV}$ is also an interminiband single-particle excitation between the lowest and the third lowest miniband. When the wave
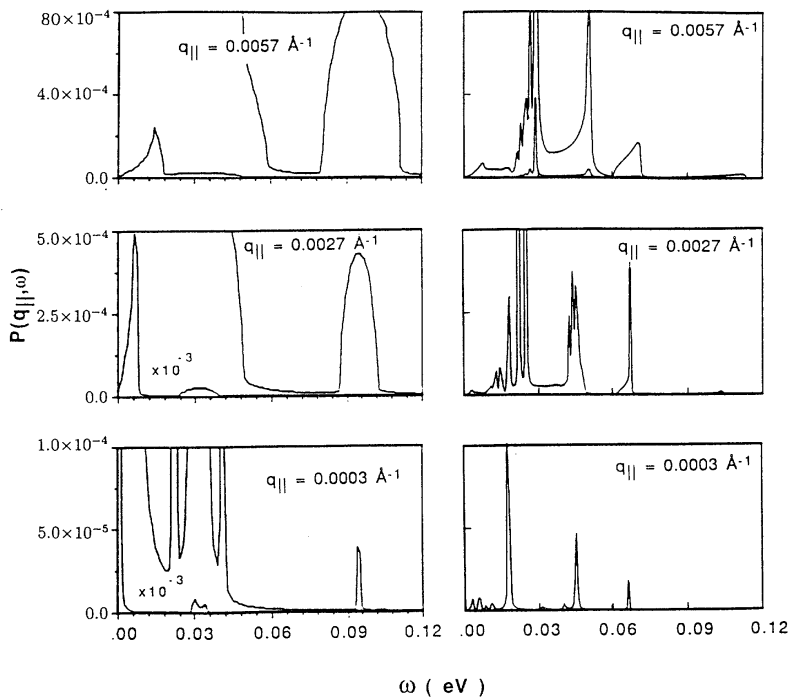

FIG. 4. Electron-energy-loss function for three $q_{\|}$values in units of $10^{4} \times 2 m^{*} e^{2} / \hbar^{2}$ for the accumulation layer $n_{s}=0.2 \times 10^{-2} \AA^{-2}, n_{D}=1.9 \times 10^{-6} \AA^{-3}, E_{B}=0.4 \mathrm{eV}, E_{s}=0.2$ $\mathrm{eV}$; the other parameters are the same as given in Fig. 1 . The left and right columns show the single-particle and collective excitations, respectively.

vector $q_{\|}$is increased, both intraminiband and interminiband single-particle excitation spectra are broadened. The collective excitation (plasmon) spectra shown in the right column are, in general, sharper than their singleparticle counterparts because of the screening effect if one takes into account the interaction between electrons. For the accumulation layer case, there are five intraminiband plasmon modes corresponding to the lowest miniband; they are all twofold degenerate because of our assumption of symmetric sample. The strongest intramini-
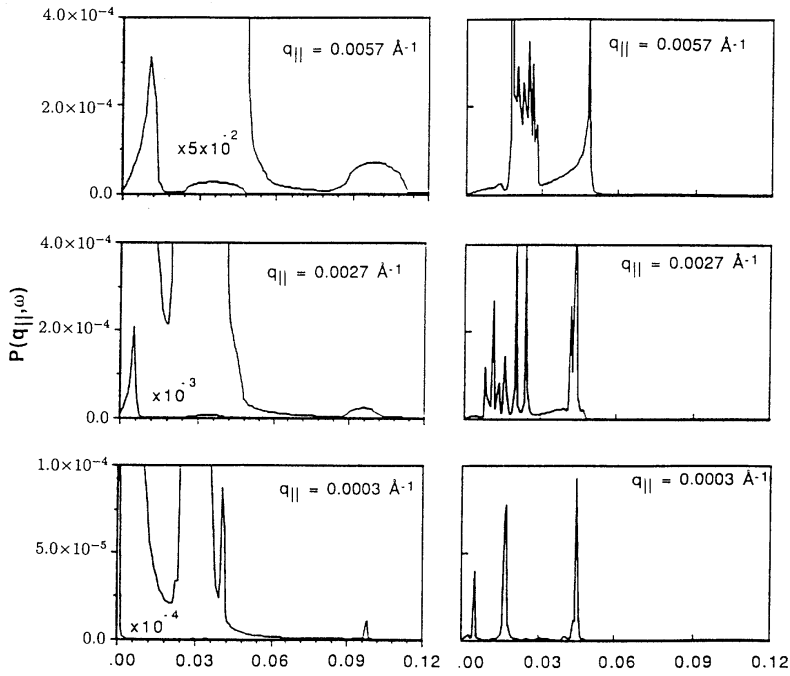

$\omega(\mathrm{eV})$ $\AA^{-2}$.

FIG. 5. $P\left(q_{\|}, \omega\right)$ for the depletion layer $n_{s}=-0.4 \times 10^{-4}$ 
band plasmon mode at $\omega \sim 0.02 \mathrm{eV}$ is the surface plasmon predicted by Giuliani and Quinn. ${ }^{5}$ Actually this mode is mainly due to the collective excitation of the electrons in the lowest Tamm state, and its strength reflects the heavy electron population while its departure from the other modes signals the spatial confinement of the Tamm state. By comparing the resonant excitation frequency with the energy gap and taking into account the depolarization effect, ${ }^{32}$ we conclude that the mode at $\omega=0.043 \mathrm{eV}$ is the interminiband plasmon associated with transitions from the lowest miniband to the empty miniband above the Fermi energy. The weak peak split off from the $0.043-\mathrm{eV}$ mode results from the weak interaction of plasmon modes from opposite surfaces. Another striking feature in Fig. 4 is the strong peak located at $\omega=0.066 \mathrm{eV}$. This mode is the intersubband plasmon excitation associated with the transitions between the heavily occupied Tamm state, which is $0.007 \mathrm{eV}$ below the lowest miniband, and the empty state above the Fermi energy. A large depolarization upshift, $\Delta_{12}=0.02 \mathrm{eV}$, about $43 \%$ of the intersubband energy difference $(\sim 0.03 \mathrm{eV})$, is observed in Fig. 4 . This large depolarization effect indicates strong Coulomb repulsion between the highly localized Tamm state and the state in the second miniband. Only in a quantum wire system where the states are quantum mechanically localized in two dimension has such a large shift been observed, both experimentally and theoretically. ${ }^{33}$ The weak peak close to $\omega=0.11 \mathrm{eV}$ is also due to an intersubband transition between the Tamm state and the empty third miniband (the interband energy difference is 0.095 $\mathrm{eV})$. As the wave vector $q_{\|}$increases, both intraminiband and interminiband spectra become broadened and their energies move upward. Single-particle excitations also contribute to the loss functions shown in the right column of Fig. 4, providing a smooth background which is strongest for larger wave vectors.

Figure 5 shows the excitation spectra corresponding to the depletion layer for which the Tamm state exists 0.01 $\mathrm{eV}$ above the lowest miniband so that it is less populated than its accumulation layer counterpart. The miniband structure remains the same as in the accumulation layer but the energy difference between the occupied Tamm state and the Fermi level is reduced to $0.018 \mathrm{eV}$. The single-particle excitation spectra for the present case are qualitatively the same as those for the accumulation layer; intraminiband excitation peaks are seen near the origin of frequency and, in addition, there are two interminiband excitation peaks near $\omega=0.03$ and $0.09 \mathrm{eV}$ belonging to the transitions between the lowest and the second and the third lowest miniband, respectively. The collective excitation spectrum shown in the right column of Fig. 5 for $q_{\|}=0.0003 \AA^{-1}$ features three peaks near $\omega=0.005,0.018$, and $0.043 \mathrm{eV}$; the latter mode is due to the interminiband plasmon excitation. The middle peak at $\omega=0.018 \mathrm{eV}$ can be interpreted by noting the energy difference between the Tamm state and the miniband above the Fermi level ( $\Delta E=0.018 \mathrm{eV})$. Unlike the accumulation layer, the depolarization effect is very small because (1) the Tamm state is much less populated, and (2) the Tamm state is weakly localized due to the mixing effect; the middle mode is nearly degenerate with the in-

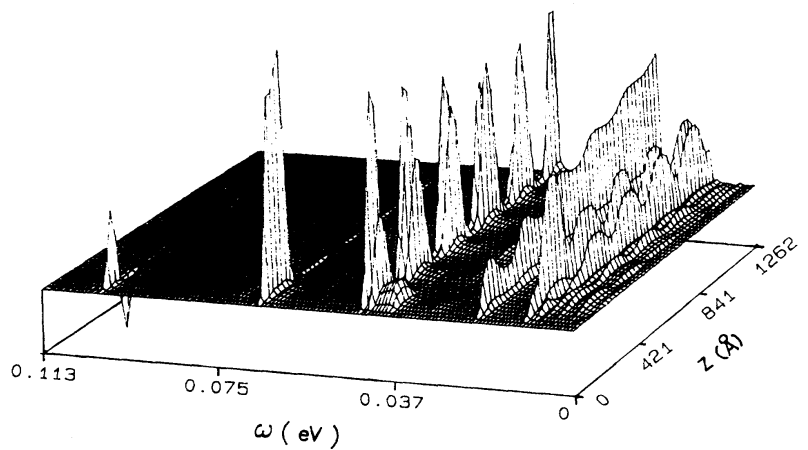

FIG. 6. The imaginary part of the induced charge density $n_{\text {ind }}\left(q_{\|}, \omega ; z\right)$, given in arbitrary units, under an external potential $\phi_{\text {ext }} \sim \exp \left(p q_{\|} z\right)$, as a function of $\omega$ and $z$ (depth inside the surface) for $q_{\|}=0.0057 \AA^{-1}$. The sample parameters are the same as in Fig. 4. The induced charge density shown includes only half of the superlattice sample.

traminiband excitation modes. When $q_{\|}=0.0027 \AA^{-1}$, the intraminiband plasmons and the interminiband plasmon due to the Tamm state grouped together to form a band. As $q_{\|}$is increased to $0.0057 \AA^{-1}$, the intraminiband plasmon band shifts upward in frequency faster than the plasmon mode due to the Tamm state, therefore, near the lower boundary a discrete plasmon mode emerges.

To support our mode assignment and to provide a microscopic visualization of the spatial localization of the plasmon modes, we calculated the induced charge density,

$$
n_{\text {ind }}\left(q_{\|}, \omega ; z\right)=\int_{0}^{L} d z^{\prime} \chi\left(q_{\|}, \omega ; z, z^{\prime}\right) \phi_{\text {ext }}\left(q_{\|}, \omega ; z^{\prime}\right),
$$

where $\chi\left(q_{\|}, \omega ; z, z^{\prime}\right)$ is the density-density response function and the external field $\phi_{\text {ext }}\left(q_{\|}, \omega ; z^{\prime}\right)$ is chosen as the Coulomb potential associated with an electron approaching the surface. The calculated induced charge densities at $q_{\|}=0.0057 \AA^{-1}$ shown in Figs. 6 and 7 for the accumulation layer and depletion layer, respectively, are rather informative. In Fig. 6, at low frequency $(\omega<0.02 \mathrm{eV})$, all the induced charge-density waves extend throughout

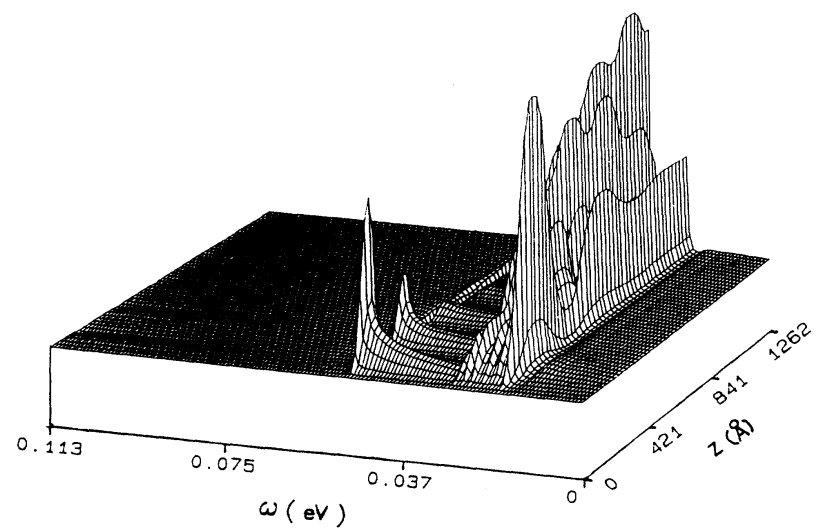

FIG. 7. The imaginary part of the induced charge density for $q_{\|}=0.0057 \AA^{-1}$; the other parameters are given in Fig. 5 . 
the superlattice and their shapes minic the wave functions. The induced charge density corresponding to the surface plasmon modes $(\omega=0.023 \mathrm{eV})$ displays a strong surface peak as well as an extension into the interior because of the interaction between the surface mode and the bulk modes. At the interminiband resonance frequency $(\omega \sim 0.043 \mathrm{eV})$, the induced charge density has its maxima at the centers of the wells, and the shape is analogous to the charge density without external probing. Two intersubband plasmon modes $(\omega=0.066,0.11 \mathrm{eV})$ due to the Tamm states exhibit unique behavior; the modes are localized within the first well which is about $260 \AA$ deep inside the surface, and the high-energy charge oscillation $(\omega \sim 0.11 \mathrm{eV})$ forms a dipole moment. In Fig. 7 near $\omega=0.018 \mathrm{eV}$, a strong mixed chargedensity oscillation pattern is demonstrated; the plasmon excitation due to the surface localized Tamm state and extended intraminiband plasmon coexist.

We now comment on the dispersion relations of the plasmon modes portrayed in Figs. 4 and 5. By scanning the maxima of the spectra we mapped out the frequency versus wave vector for the plasmon modes; the results for the accumulation and depletion layers are given in Figs. 8 and 9. The lowest mode indicates the upper edge of particle-hole excitation of the lowest Tamm state. Four discrete intraminiband plasmon modes (twofold degenerate) form a plasmon band at nonzero wave vector $\left(q_{\|}=0\right) .{ }^{34}$ The dashed lines show the upper and lower limit of the plasmon band of a cyclic superlattice predicted by Giuliani and Quinn, ${ }^{5}$ and the limits match our re-

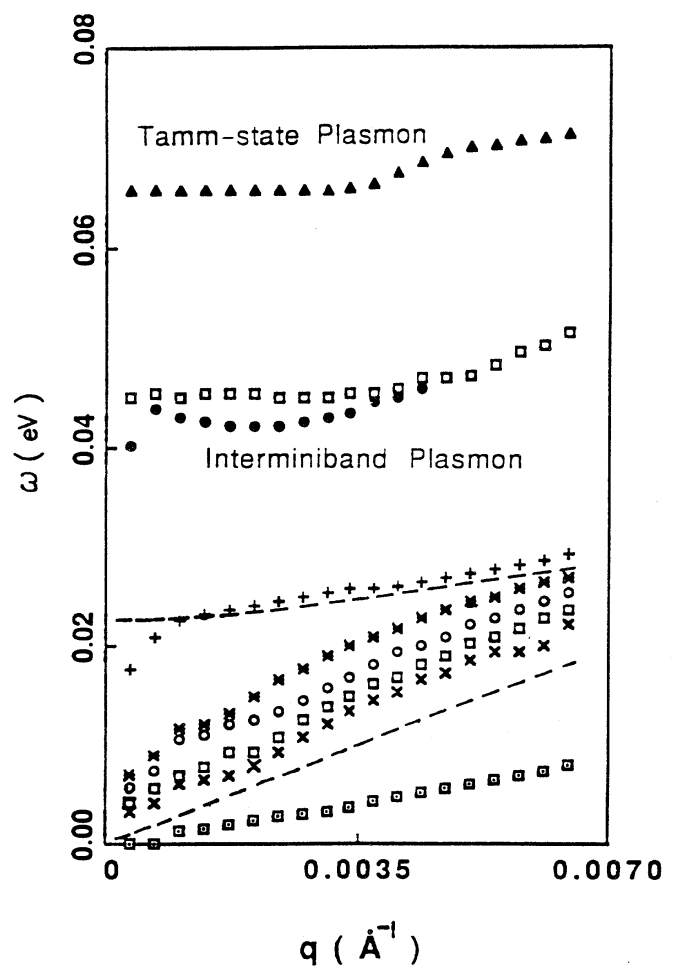

FIG. 8. Plasmon dispersion obtained by scanning the maxima of the loss function $P\left(q_{\|}, \omega\right)$ shown in Fig. 4. The dashed lines are the predictions of Giuliani and Quinn (Ref. 5).

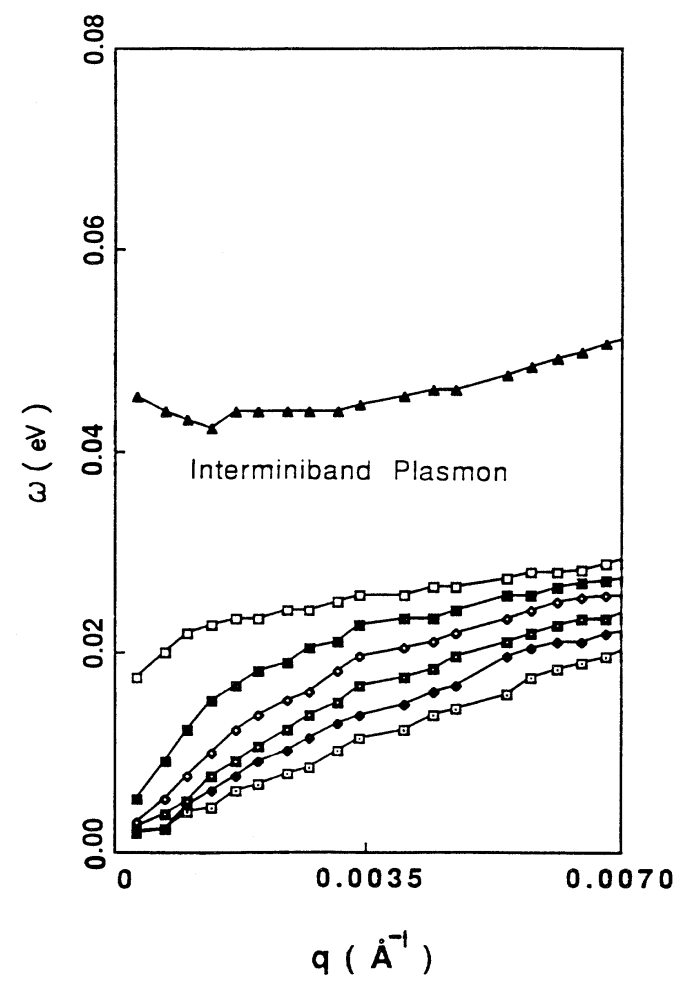

FIG. 9. Plasmon dispersion relations corresponding to the loss functions shown in Fig. 5.

sult fairly well. The surface plasmon mode indicated by the plus sign is due to the Tamm state which splits off the plasmon band and varies as $q_{\|}^{1 / 2}$ for small $q_{\|}$suggesting that the electron gas inducing the Tamm state is quasitwo-dimensional; recall the characteristic dispersion relation of a two-dimensional electron gas, $\omega \sim q_{\|}^{1 / 2}$. This plasmon mode remains strong and narrow (Landau damping free) until it crosses the single-particle excitation continuum of the electrons in the lowest Tamm state at very large wave-vector transfer, $q_{\|}=0.02 \AA^{-1}$. The second band above $0.04 \mathrm{eV}$, an interminiband plasmon band, has a finite width for small $q_{\|}$because of the coupling between the plasmon modes for different eigenstates; the plasmon bandwidth is about $0.004 \mathrm{eV}$, very close to the sum of the lowest miniband widths, $\Delta_{1}+\Delta_{2}=0.0049 \mathrm{eV}$. The strong interaction of the plasmon modes depresses the lowest interminiband plasmon mode such that its dispersion becomes negative. The plasmon mode due to the Tamm state is not dispersive for small $q_{\|}$, until the wave vector $q_{\|}$is greater than $0.0035 \AA^{-1}$, at which the probing depth $\left(\sim q_{\|}{ }^{-1} \sim 290 \AA\right)$ is less than the localization length of the state (300 $\AA$ ). This plasmon mode is free of Landau damping until $q_{\|}=0.015 \AA^{-1}$, and at still larger $q_{\|}$values $\left(q_{\|} \geq 0.018\right.$ $\AA^{-1}$ ) the plasmon mode merges with the particle-hole excitation continuum to form a broad background. Figure 9 shows the dispersion relations for the depletion layer. In the present case, the frequency of the plasmon mode due to the Tamm state is lower than the plasmon band. The interminiband plasmon dispersion appears approxi- 
mately the same as that associated with the accumulation layer as one expected because of the similarity of the minibands for both cases.

In HREELS, the detector collects essentially all the inelastically scattered electrons within an aperture of a few degrees. To make our calculated HREELS spectrum directly comparable with experiment, we have carried out the integration of $P\left(q_{\|}, \omega\right)$ over $q_{\|}$, which yields the scattering probability per unit frequency in the specular direction with energy loss $\hbar \omega$, using the formula given by Camley and Mills. ${ }^{35}$ Because of the high strength and weak dispersion of the plasmon mode noted above, a very well-defined feature peaked at $\omega=0.069 \mathrm{eV}$ can be seen after the integration, suggesting that this plasmon mode should be measurable in the HREELS experiment. The intensity of the energy-loss peak increases with decreasing impact energy (decreasing probe depth) because of the kinematic effect as well as the nature of the surface localization of the mode. We have also found that another way to enhance the loss intensity is to increase the angle of incidence (i.e., decreases the probe depth). The details of these studies will be included in another publication.

\section{CONCLUSIONS}

In conclusion, we have proposed a new self-consistent approach to calculate the electronic level structure of a semiconductor superlattice of a finite number of layers. The electronic miniband structure has been reproduced and the surface localized states (Tamm states) split off from and existing above and below the miniband continuum have been found. One of the Tamm states found crosses the Fermi energy as the surface depletion effect increases. We have calculated the electronic eigenstates of a superlattice as a function of the barrier height difference $\Delta E$ between the internal surface and the interior barriers. An excellent agreement with light-absorption data has been achieved within our self-consistent framework for the case of higher internal surface barrier $(\Delta E>0)$. We found a Tamm state below the miniband continuum for lower internal surface barrier $(\Delta E<0)$. The dynamical properties of a superlattice have been investigated theoretically. Interminiband and intraminiband plasmons have been studied. We have also found a plasmon mode due to the Tamm state. We proposed the use of HREELS to detect this plasmon mode.

\section{ACKNOWLEDGMENTS}

We acknowledge useful discussions with S. E Ulloa. We are grateful to J. C. Hermanson for stimulating discussions and his critical reading of the manuscript. The work was supported in part by the Faculty Research Fund of Central Washington University.

${ }^{1}$ For recent review, see, for example, L. Easki, IEEE J. Quantum Electron. QE-22, 1611 (1986); G. Bastard, J. A. Brum, and R. Ferreira, in Solid State Physics, edited by H. Ehrenreich and D. Turnbull (Academic, New York, 1991), Vol. 44.

${ }^{2}$ R. Dingle, A. C. Gossard, and W. Wiegmann, Phys. Rev. Lett. 34, 1327 (1975)

${ }^{3}$ D. Olego, A. Pinczuk, A. C. Gossard, and W. Wiegmann, Phys. Rev. B 25, 7867 (1982); A. Pinczuk, M. G. Lamont, and A. C. Gossard, Phys. Rev. Lett. 56, 2092 (1986).

${ }^{4}$ G. Fasol, N. Mestres, H. P. Hughes, A. Fisher, and K. Ploog, Phys. Rev. Lett. 56, 2517 (1986).

${ }^{5}$ G. F. Giuliani and J. J. Quinn, Phys. Rev. Lett. 51, 919 (1983).

${ }^{6}$ H. L. Störmer, J. P. Eisentein, A. C. Gossard, W. Wiegmann, and K. Baldwin, Phys. Rev. Lett. 56, 85 (1986).

${ }^{7}$ H. Ohno, E. E. Mendez, J. H. Brum, J. M. Hong, F. AgulloRueda, L. L. Chang, and L. Easki, Phys. Rev. Lett. 64, 2555 (1990).

${ }^{8}$ I. Tamm, Phys. Z. Sowjetunion. 1, 733 (1932).

${ }^{9}$ D. L. Smith and C. Mailhiot, Rev. Mod. Phys. 62, 173 (1990).

${ }^{10}$ G. Danan, B. Etienne, F. Mollot, R. Planel, A. M. Jean Louis, F. Alexandre, B. Jusseraud, G. Le Roux, J. Y. Marzin, H. Savary, and B. Sermage, Phys. Rev. B 35, 6207 (1987); K. J. Moore, G. Duggan, P. Dawson, and C. T. Foxon, ibid. 38, 5535 (1988).

${ }^{11}$ J. N. Schulman and Y. C. Chang, Phys. Rev. B 31, 2056 (1985); J. Ihm, Appl. Phys. Lett. 50, 1068 (1987); Y. T. Lu and L. J. Sham, Phys. Rev. B 40, 5567 (1989).

12J. Zhang and S. E. Ulloa, Phys. Rev. B 38, 2063 (1988).

${ }^{13}$ M. Steslicka, R. Kocharczyk, and M. L. Glasser, Phys. Rev. B 42, 1458 (1990).

${ }^{14}$ W. L. Boss, Phys. Rev. B 44, 8035 (1991).

${ }^{15}$ S. G. Tikhodeev, Solid State Commun. 78, 339 (1991).

16J. Zhang, S. E. Ulloa, and W. L. Schaich, Phys. Rev. B 43, 9865 (1991).

${ }^{17}$ J. Zhang, S. E. Ulloa, and W. L. Schaich, Phys. Rev. B 41, 5467 (1990).

${ }^{18}$ P. Apell, Phys. Scr. 24, 795 (1981); P. J. Feibelman, Prog. Surf. Sci. 12, 287 (1982); P. Appel, A. Lyungbert, and S. Lundqvist, Phys. Scr. 30, 367 (1984).

${ }^{19}$ F. Y. Huang and H. Morkoc, J. Appl. Phys. 71, 524 (1992).

${ }^{20}$ D. H. Ehlers and D. L. Mills, Phys. Rev. B 36, 1051 (1987).

${ }^{21}$ S. R. Streight and D. L. Mills, Phys. Rev. B 38, 8526 (1988).

${ }^{22}$ S. R. Streight and D. L. Mills, Phys. Rev. B 40, 10488 (1989).

${ }^{23}$ R. H. Yu and J. C. Hermanson, Phys. Rev. B 40, 11851 (1989).

${ }^{24}$ R. H. Yu and H. C. Hermanson, Phys. Rev. B 41, 5991 (1990).

${ }^{25}$ D. Aitelhabti, P. Vasilopoulos, and J. F. Currie, Can. J. Phys. 68, 268 (1990).

${ }^{26}$ L. Hedin and B. I. Lundqvist, J. Phys. C 4, 2064 (1971).

${ }^{27}$ A. G. Eguiluz, Phys. Rev. B 31, 3303 (1985).

${ }^{28}$ Y. Joe and S. E. Ulloa, J. Phys. Condens. Matter 2, 7137 (1990).

${ }^{29}$ F. Agullo-Rueda, E. E. Mendez, H. Ohno, and J. M. Hong, Phys. Rev. B 42, 1470 (1990).

${ }^{30}$ D. Gammon, B. Shanabrook, J. Ryan, G. Brozak, and D. Katzer, Surf. Sci. 263, 474 (1992).

${ }^{31}$ L. Brey, N. Johnson, and B. Halperin, Phys. Rev. B 40, 10647 (1989).

${ }^{32}$ D. A. Dahl and L. J. Sham, Phys. Rev. B 16, 651 (1977).

${ }^{33}$ W. Hansen, M. Horst, J. P. Kotthaus, U. Merkt, Ch. Sikorski, and K. Ploog, Phys. Rev. Lett. 58, 2586 (1987); T. Demel, D. Heitmann, P. Grambow, and K. Ploog, Phys. Rev. B 38, 12732 (1988); W. M. Que and G. Kirczenow, ibid. 37, 7153 (1988); Q. Li and S. Das Sarma, ibid. 40, 5860 (1989); R. H. Yu and J. C. Hermanson, ibid. 42, 1496 (1990).

${ }^{34}$ J. K. Jain, and P. B. Allen, Phys. Rev. Lett. 54, 2437 (1985).

${ }^{35}$ R. E. Camley and D. L. Mills, Phys. Rev. B 37, 10378 (1988); 29, 1696 (1984). 